\title{
Fibroblasts orchestrate tumour initiation
}

Colorectal cancers (CRCs) originate from epithelial stem cells in intestinal crypts but whether the surrounding mesenchymal cells contribute to tumorigenesis is not known. Roulis, Kaklamanos et al. conducted single cell RNA sequencing (scRNA-seq) in mice to characterize intestinal mesenchymal cells and in doing so found a population of rare fibroblasts present in intestinal crypts that promote intestinal tumorigenesis in a paracrine manner.

Several populations of fibroblasts were identified through scRNA-seq, and pathway analyses of these cells indicated that two populations had enrichment of arachidonic acid metabolism. This pathway is strongly associated with CRC: inhibition of prostaglandin-endoperoxide synthase 2 (PTGS2, also known as cyclooxygenase 2 (COX2)), an enzyme in the pathway, prevents CRC, although the molecular mechanism for this is not known. Immunostaining in mouse intestines indicated that one of the fibroblast populations with high PTGS2 levels was a population known as Cajal cells in the muscular layer. The second was a rare population located close to the stem cell zone of intestinal crypts; the authors termed these cells rare pericryptal PTGS2-expressing fibroblasts (RPPFs).

Fibroblast-specific knockout of Ptgs 2 in mice indicated that fibroblasts are the predominant source of PTGS2 in intestines. To determine the role of this pathway in tumour formation, $A p c^{\mathrm{Min} /+}$ mice (which spontaneously develop intestinal tumours) were crossed with mice lacking Ptgs 2 in fibroblasts. These $A p c^{\mathrm{Min} /+} ;$ Ptgs $2^{\Delta \mathrm{Fibr}}$ mice had a reduction in tumour formation, but there was no difference in the size of tumours that did form, indicating that PTGS2 in fibroblasts is important for tumour initiation but not growth. Similar results were observed using two additional mouse models: one in which Ptgs 2 expression in $A p c^{\mathrm{Min} /+}$ mice was induced exclusively in fibroblasts and one in which intestinal tumorigenesis was driven by injections of the mutagen azoxymethane in Ptgs $2^{\Delta \text { Fibr }}$ mice.

Lipidomic analyses indicated that there was a reduction of prostaglandin $\mathrm{E} 2\left(\mathrm{PGE}_{2}\right)$ and prostaglandin $\mathrm{I} 2\left(\mathrm{PGI}_{2}\right)$ secretion in intestines of Ptgs $2^{\Delta \mathrm{Fibr}}$ mice compared with control mice. $\mathrm{PGE}_{2}$ has a known tumour-promoting role, so the authors hypothesized that fibroblast-derived $\mathrm{PGE}_{2}$ might be responsible for intestinal tumour initiation in their mouse models.

To look more closely at the role of $\mathrm{PGE}_{2}$, the authors cultured intestinal crypts from wild-type mice in organoid growth media (OGM) in the presence of exogenous $\mathrm{PGE}_{2}$. This led to the growth of spheroids rather than typical budding organoids with a crypt-like structure, indicating a lack of differentiation and increased stem cell-like behaviour. They determined that the key receptor for $\mathrm{PGE}_{2}$ in intestinal epithelial cells was PTGER4, and OGM cultures of crypts lacking Ptger4 in intestinal epithelial cells (Ptger $^{\triangle \mathrm{IEC}}$ ) remained differentiated in the presence of $\mathrm{PGE}_{2}$. Similarly, spheroids grew when control but not Ptger $4^{\triangle \mathrm{IEC}}$ crypts were co-cultured with fibroblasts in OGM. Spheroid formation was also blocked by a PTGER4 inhibitor. scRNA-seq supported the increased stem cell function of epithelial cells in response to signalling through PTGER4; importantly, this pathway induced expansion of a population of cells that typically functions as reserve stem cells (RSCs).
PTGER4 activation induced transcription of YAP1 target genes, and deletion of Yap1 from intestinal crypts (Yap1 $1^{\Delta \mathrm{IEC}}$ crypts) prevented spheroid formation in the presence of fibroblasts in OGM. Furthermore, RSC expansion was prevented in Yap $1^{\triangle \mathrm{IEC}}$ crypt and fibroblast co-cultures. Stimulation of wild-type organoid cultures with $\mathrm{PGE}_{2}$ induced YAP1 dephosphorylation, nuclear translocation and downstream transcription, which were blocked by a PTGER4 inhibitor. YAP1 nuclear localization was also observed in early stage adenomas in $A p c^{\mathrm{Min} /+}$ mice, as was expansion of a population of cells expressing SCA1 (an RSC marker and YAP1 target gene). This was not observed in $A p c^{\mathrm{Min} /+} ; Y_{a p 1} 1^{\Delta \mathrm{IEC}}$ mice or $A p c^{\mathrm{Min} /+} ;$ Ptger $4^{\triangle \mathrm{IEC}}$ mice, and these mice developed fewer tumours. As with the authors' earlier mouse models, the few tumours that did form did not have reduced growth, again indicating that tumour initiation, but not growth, is affected by this pathway.

The authors demonstrated that organoid cultures of crypts isolated from normal human colon tissue formed spheroids in the presence of $\mathrm{PGE}_{2}$, and this was blocked by a PTGER4 inhibitor. Furthermore, YAP1 immunostaining in tissues from patients with colorectal tumours indicated that YAP1 is nuclear in tumours but not neighbouring normal tissue.

This study has illuminated a fibroblast-driven molecular pathway by which tumour initiation might occur. As COX2 inhibitor toxicity has prevented their use in cancer prevention and PTGER4 inhibitors are available, it will be especially interesting to determine whether PTGER4 inhibitors might be used for preventing CRC development.

Sarah Seton-Rogers 